\title{
Philosophiques
}

\section{L'esthétique de Dilthey : phénoménologie et théorie littéraire}

\section{Peter McCormick}

Volume 2, numéro 2, octobre 1975

URI : https://id.erudit.org/iderudit/203032ar

DOI : https://doi.org/10.7202/203032ar

Aller au sommaire du numéro

Éditeur(s)

Société de philosophie du Québec

ISSN

0316-2923 (imprimé)

1492-1391 (numérique)

Découvrir la revue

Citer cet article

McCormick, P. (1975). L’esthétique de Dilthey : phénoménologie et théorie littéraire. Philosophiques, 2(2), 229-252. https://doi.org/10.7202/203032ar d'utilisation que vous pouvez consulter en ligne.

https://apropos.erudit.org/fr/usagers/politique-dutilisation/ 


\title{
L'ESTHÉTIQUE DE DILTHEY : PHÉNOMÉNOLOGIE ET THÉORIE LITTÉRAIRE *
}

\author{
par Peter McCormick
}

Depuis la cristallisation d'une théorie de l'interprétation dans le chef-d'œuvre de H.G. Gadamer, Wabrbeit und Mebode, la théorie littéraire de Dilthey a soulevé un nouvel intérêt. ${ }^{1}$ Pendant plusieurs années, elle a été négligée ${ }^{2}$ à cause des attaques de toute une école de critiques allemandes dont les principaux théoriciens étaient Viëtor, Kayser et Staiger. On accusait Dilthey d'exagérer les aspects historiques et psychologiques des textes au détriment de leur caractère formel. L'œuvre de Gadamer a éclairé les enseignements, aujourd'hui oubliés, que Dilthey avait reçus au cours de son travail exhaustif sur Schleiermacher. La publication récente de la première bibliographie complète sur

* Une ébauche plus courte de ce texte a été prononcée au $44 \mathrm{e}$ Congrès de l'Association canadienne-française pour l'avancement des sciences à Moncton, au Nouveau-Brunswick, le 7 mai 1975.

1. Tübingen, Mohr, 1960. La deuxième édition (1965) contient une longue réponse aux critiques de la première édition. Voir aussi: GADAMER, Kleine Scbriften, Tübingen, Mohr, I (1967), II (1968), III (1972), et IV (à paraître). La direction particulière du travail de Gadamer sur Dilthey peut être retracée dans "Portée et limites de l'œuvre de Wilhelm Dilthey» dans son livre Le Probleme de la conscience bistorique, Louvain, 1963, pp. 21-37.

2. Voir: VIËTOR, «Deutsche Literaturgeschichte als Geistesgeschichte: Ein Rückblick », PMLA, LX (1945), 899-916, un article qui fait autorité. Pour le point de vue de W. KAYSER, voir son texte connu: Das spracblicbe Kunstwerk, Berne, 6e édition, 1960. Les opinions de STAIGER sont dispersées. Un résumé convenable se trouve dans son Grundbegriffe der Poetik, Zurich, 4e édition, 1949, un livre également connu. WELLEK et WARREN, dans leur Theory of Literature, New York, 1956, suivent de près ce courant allemand. 
Dilthey, ${ }^{3}$ accompagnée d'une étude intéressante en allemand sur son esthétique, ${ }^{4}$ montre clairement que les stéréotypes auxquels on avait réduit la pensée de Dilthey ne sont plus acceptables.

À la lumière donc de ces travaux, et surtout de celui de Müller Vollmer, ${ }^{5}$ je me bornerai dans cet article à une discussion critique de quatre points : la critique diltheyenne de la tradition esthétique, la thèse cardinale de sa propre théorie, sa classification des énoncés et, enfin, ses concepts d'Erlebnis et du Typ. Mais, d'abord, « la théorie littéraire de Dilthey, » à quoi se réfère-t-elle?

\section{I - La «théorie littéraire» de Diltbey}

Le premier problème pour réussir à présenter un exposé précis et constructif de la «théorie littéraire de Dilthey (disons la «théorie 》) est de décider si une telle phrase se réfère à un contenu réel et distinct. Il nous faut, en effet, une distinction entre les vues de Dilthey et les écrits très divergents des théoriciens de la geistesgeschiechtliche école: F. Gundolf, R. Unger,

3. HERRMANN, U., Bibliograpbie Wilbelm Dilthey, Weinheim, 1969. Cette bibliographie est complète jusqu'à l'été 1968. Elle fournit une liste chronologique de toutes les publications de Dilthey, de tous ses plans, ses lectures, ses lettres, son journal et ses écrits posthumes. Elle fournit également une liste des traductions dans six langues. La liste chronologique détaillée des écrits de Dilthey lui-même couvre 90 pages, alors que la liste des écrits secondaires couvre 84 pages. De Dilthey, seuls le Einleitung de 1833 et les tomes V, VI et VIII des Oeuvres complètes (Gesammelte Schriften ou GS) ont été traduits en français.

4. RODI, F., Morpbologie und Hermeneutik: Zur Metbode von Diltheys Aestbetik, Stuttgart, 1969. Pour un compte rendu plus général de l'œuvre de Dilthey, du point de vue allemand contemporain, voir la longue introduction de M. RIEDEL à l'édition augmentée du livre de Dilthey Der Aufbau der geschicbtlichen Welt in den Geisteswissenschaften, Francfort, 1970, pp. 9-81, et les articles antérieurs, plus spécialisés, du même auteur: "Wilhelm Dilthey und das Problem der Metaphysik », Pbil. Jabrb. 76 (1968/69), 332-348, et "Das erkenntniskritische Motiv in Diltheys Theorie der Geisteswissenchaften », Hermeneutik und Dialektik, vol. I, Tübingen, 1970, pp. 233-257. Beaucoup plus ancien (1933), l'ouvrage de O.F. BOLLNOW, Diltbey: Eine Einführung in seine Pbilosopbie, Stuttgart, 2e édition, 1955, demeure très utile. En anglais, The Pbilosopby of W. Diltbey, Londres, 1952, de H. HODGES demeure également utile. 
O. Walzel, et J. Petersen. ${ }^{6}$ Voici une schématisation de la présentation de Müller-Volmer.

(1) « La 'théorie' correspond à cette partie des écrits de Dilthey qui traitent spécificuement de la littérature. » C'est l'opinion générale autant des disciples de Dilthey que de ses critiques. Pourtant, ce point de vue est inadéquat pour deux raisons. En pratique, ce point de vue se concentre uniquement sur trois ouvrages, à l'exclusion de plusieurs ouvrages antérieurs de Dilthey sur la littérature. ${ }^{7}$ De plus, ce point de vue nie tout lien entre les aspects littéraires et les autres aspects (principalement historiques et philosophiques) de son œuvre. Mais cette dénégation est inutile car ce lien est indéniable.

(2) « La 'théorie' est identique à la théorie littéraire contenue dans toute l'ouvre de Dilthey.» Cette assertion est aussi inadéquate pour deux raisons. De fait, une lecture exhaustive de l'œuvre de Dilthey montre qu'il n'y a aucun endroit où cette théorie littéraire puisse être appréhendée dans sa totalité. En outre, quelques-unes des différentes présentations de la théorie littéraire sont inconsistantes.

(3) «La 'théorie' est identique à la théorie littéraire qui peut être déduite de la ptatique littéraire critique de Dilthey. » Cette opinion est inadéquate à cause de la deuxième raison citée plus haut sous (1).

(4) «La 'théorie' est identique à l'établissement des contributions terminologiques durables de Dilthey (exemple: Bildungsroman, génération littéraire). $\gg$ Cette opinion est également inadéquate parce qu'il y a mésentente sur le choix des critères

5. Towards a Pbenomenological Theory of Literature: A Study of W. Diltbey's Poetik, La Haye; 1963. Les numéros des pages dans le texte se réfèrent à ce livte.

6. GUNDOLF, F., Goethe, Berlin, 1916; UNGER, R., Herder, Novalis und Kleist, Francfort, 1922 ; WALZEL, O., Gebalt und Gestalt im Kunstwork des Dichters, Postdam, 1923; et PETERSEN, J., Die Wissenscbaft von der Dichters, Berlin, 1939.

7. Ces ouvrages sont trois recueils d'essais: le célèbre Das Erlebnis und die Dichtung (1905), Von Deutscber Dichtung und Musik (posthume, 19572), et Die grosse Phantasiedichtung und andere Studien $z u$ vergleichenden Literaturgescbichte (posthume, 1954). 
pour définir cet établissement et sur les termes auxquels se rapportent ces critères. Ainsi, il semblerait que la «théorie » n'ait aucun référent identifiable.

La propre proposition de Müller-Vollmer (pp. 45-46) se lit comme suit :

(5) la «théorie » est identique à l'essai Die Einbildungskraft des Dicbters (1887) de Dilthey, à sa suite Die drei Epochen der modernen Aesthetik und zhre beutige Aufgabe (1892), aux parties de quatre autres ouvrages, ${ }^{8}$ et aux opinions contenues passim dans des parties d'ouvrages postérieurs à $1900{ }^{9} \mathrm{Il}$ écrit : « la clé de la théorie de la littérature... est contenue dans les études philosophiques écrites dans la dernière décade de sa vie, au cours de laquelle il lutta pour la clarification et la systématisation d'opinions et d'intuitions antérieures» (p. 43). Pour MüllerVollmer la valeur de cette clé, c'est qu'elle procure un moyen de dénier les arguments cités ci-haut $((1)$ - (4)). En effet, le cinquième propos en viendrait à stipuler que l'essentiel de la «théorie » n'est nullement repérable dans les écrits littéraires de Dilthey. Mais plutôt que la «théorie » doit être surtout décelable dans la combinaison des derniers écrits philosophiques et, précisément, de ces écrits littéraires (les essais de 1887 et 1892) dans lesquels... « l'éclair survint à travers une nouvelle perspective philosophique qui aboutit aux écrits phénoménologiques des années postérieures à 1900 »(p. 44). S'appuyant sur les remarques de G. Misch en 1923 et celles de L. Landgrebe en $1928,{ }^{10}$ Müller-Vollmer considère comme établi qu'il y ait eu un tel

8. Ideen «über eine besibriebende und zergliedernde Psychologie: 1896 (GS, V. 241-316). Einleitung in die Geisteswissenscbaften: 1883 (GS, I. 3-429) et la monographie Das Leben Scbleiermacbers: 1867-1870.

9. Pour ces ouvrages, voir la liste complète dans Herrmann (cf. note 3).

10. Müller-Vollmer considère les interprétations de G. Misch et de L. Landgrebe comme une semence. L'Introduction de G. MISCH au cinquième tome des Oeuvres completes de Dilthey ("Vorbericht des Herausgebers», in GS. V. Göttingen, 1914, 7-117) et son article plus récent («Die Idee der Lebensphilosophie in der Thcorie der Geisteswissenschaften, 》 Kant-Studien XXXI (1926), 536-48) doivent être nettement distingués de son livre très dense, Lebenspbilosopbie und Pbänomenologie: Eine Auseinanderstezung der Diltbeyschen Richtung mit Heidegger und Husserl, Leipzig, 1931. L'excellent article de L. LANDGREBE est paru dans le Jabrbuch de Husserl, «Wilhelm Dilthey's Theorie der Geisteswissenschaften: Analyse ihter Grundbegriff, 》 Jabrbuch für Pbil. u. phän. Forscbung, IX, (1928), 238-366. 
éclair. Mais, même si c'était vrai, nous n'avons jusqu'ici qu'une possibilité de justification de la première partie du cinquième propos. Müller-Vollmer offre-t-il une justification pour les autres parties? La réponse simple est «non» (p. 46). Et, en conséquence, cette omission constitue une importante faiblesse de l'ouvrage de Müller-Vollmer.

Il faut ajouter un argument positif à notre conclusion négative dans le fait que le cinquième propos évite les désavantages des quatre premiers. Müller-Vollmer maintient que la «théorie» doit inclure au moins quelques ouvrages philosophiques de Dilthey, parce que «les théories littéraires ont été essentiellement et par nécessité philosophiques » (p. 46). Cela suppose que chercher la «théorie » en dehors des ouvrages philosophiques serait négliger la vérité de cette assertion. Mais il est maintenant évident qu'il ne faille trouver que dans les ouvrages philosophiques les caractères essentiels et nécessairement philosophiques de la «théorie ». En fait, l'argument positif de Müller-Vollmer n'est pas convaincant, parce qu'il assume, sans preuve suffisante, que le caractère philosophique de la «théorie» se trouve surtout dans les ouvrages philosophiques.

Nous en revenons donc à l'argument négatif. Mais il n'est pas plus convaincant, parce qu'il assume que Misch et Landgrebe sont justes dans leur interprétation du développement philosophique de Dilthey et que cet éclair privilégie, pour la recherche de la «théorie», le champ des derniers écrits philosophiques. Pour la suite de l'argumentation, acceptons la première supposition. La seconde doit être démontrée, ce que l'auteur ne fait pas. Or le cinquième énoncé, la solution proposée par MüllerVollmer aux difficultés des quatre premiers, est inadéquate, parce que l'hypothèse supposant que l'éclair philosophique du Poetik de 1887 élit cet ouvrage parmi d'autres comme une base suffisante pour interpréter la théorie littéraire de Dilthey, se montre à la fois insuffisante et non convaincante. Voici une difficulté plus importante de l'étude de Müller-Vollmer: il ne parvient pas à résoudre le problème extrêmement important qu'il a luimême soulevé. Néanmoins, que son livre reste l'étude la plus 
valable, en anglais, ${ }^{11}$ fait honneur à la persistence de l'attention qu'il a portée aux ouvrages volumineux de Dilthey.

\section{II - Dilthey et la tradition estbétique}

Avant de scruter les éléments centraux de la théorie littéraire chez Dilthey, il serait utile de rappeler le but de la poétique de Dilthey, ses critiques des théories traditionnelles et les points essentiels de son propre point de vue.

Quel était le but de la poétique de Dilthey? Du point de vue avantageux de ses larges intérêts personnels, Dilthey a constaté en 1880 qu'une rupture de communications se produisait entre le public et les artistes, les artistes et les critiques, les critiques et les philosophes. Il ne fallait pas ignorer cette rupture. Pour que le travail de l'artiste soit plus facilement compris par son auditoire, il devrait faire appel à l'aide de ses critiques. Et si les critiques, de leur côté, devaient empêcher leurs propres confusions conceptuelles d'alourdir leur tâche d'interprétation, ils devraient faire appel à l'aide des philosophes. En outre, cette rupture indiquait l'échec des anciennes théories esthétiques. Les canons esthétiques classiques et postclassiques n'étaient plus applicables à l'intérêt marqué des modèles pour les problèmes sociaux et politiques, pour la «véracité » et la sincérité, pour l'expérience fascinante et minutieuse de nouvelles techniques. La tâche de Dilthey a donc été d'élaborer une nouvelle esthétique pour les modernes. Sa première question était la suivante: «considérant la désuétude des théories traditionnelles d'esthétique et l'absence de principes d'évaluation universellement reconnus, comment le philosophe pourrait-il établir la « relation naturelle » entre la théorie esthétique et la création artistique?» (p. 56). En effet, Dilthey devait élaborer sa réponse à cette question à l'intérieur d'un champ critique très précis des anciennes approches à l'esthétique, une critique que Gadamer devait utiliser plus tard dans sa Wabrbeit und Metbode.

11. Malgré quelques impertinences, l'article de R. WELLEK demeure le meilleur en anglais - "Wilhelm Dilthey's Poetics ad Literary Theory, Wäcbter und Hüter, New Haven, 1957, pp. 121-132. En ce qui concerne les livres, il faut ajouter le texte de R. MAKKREEL, Dilthey: Philosopher of the Human Sciences, qui est sous presse. 
Quelles étaient ces critiques fondamentales des théories esthétiques précédentes?

Pendant qu'il se confinait aux théories de la postRenaissance, Dilthey proposa de distinguer trois principaux types de théorie: une théorie rationaliste, une théorie psychologiste et la théorie idéaliste allemande.

La théorie rationaliste, d'abord introduite par les théories cartésiennes de Boileau et d'Aubignon, aboutit au système de Leibniz. Ce système devait compter des adeptes jusqu'à Mendelssohn et Lessing. Dilthey croyait que l'essentiel de cette théorie résidait dans l'utilisation leibnizienne des notions de force et d'instinct pour expliquer les représentations mentales, et dans la conception leibnizienne de l'harmonie cosmique. L'art était devenu une «présentification sensorielle de l'harmonie dans l'ordre du monde» (sinnliche Vergegenwärtigung des barmonischen Weltzusammenbangs). Le problème de cette théorie était qu'elle réduisait les impressions esthétiques à des corrélations rationnelles entre l'esprit et le cosmos. Toutefois, cet accent rationaliste mis sur des normes constitutives pour tout art a donné à Dilthey l'une des clés de son propre système.

Les théories psychologistes du siècle suivant ont été dérivées, d'après Dilthey, des méthodes empiriques. Cette approche aboutit à Elements of criticism (1762) de H. Home, la source de l'esthétique expérimentale moderne. Dilthey croyait que ce livre avait effectivement ouvert la voie aux théories de Kant en proposant l'identification des impressions esthétiques aux qualités objectives définies. Ici, deux idées fondamentales s'imposent. D'abord, puisqu'on peut distinguer le plaisir qu'une personne tire d'un certain objet et le désir qu'elle en éprouve, on peut identifier l'expérience esthétique au désintéressement. Deuxièmement, l'objet esthétique, en étant la «présence idéelle» d'un objet, peut dissocier le plaisir et le désir. Dilthey a cru que l'ouvrage de Home sur l'analyse descriptive et la corrélation entre les impressions émotives et les qualités objectives particulières établissait un cadre méthodologique pour traiter de l'expérience esthétique. Néanmoins, Dilthey a soulevé un certain nombre de griefs contre la psychologie des impressions esthétiques. En voici quelques-uns: la circularité de l'interprétation psychologiste, la 
distorsion sur laquelle opèrent les méthodes empiriques, les aspects du conditionnement historique des expériences esthétiques et, à travers l'analyse, la déviation nécessaire d'un phénomène sympathique en impressions diverses.

Dans le troisième et dernier groupe de théories, celui du classicisme et du romanticisme allemands, Dilthey a pu mettre en jeu le savoir extraordinaire qu'il avait acquis pendant la période prolifique de son étude herméneutique sur Schleiermacher. ${ }^{12}$ Il n'est donc pas surprenant que Dilthey ait vu l'échec définitif des théories objectivistes aristotéliciennes de la mimesis dans l'apogée du tournant subjectiviste que l'esthétique rationaliste et analytique avait déjà pris dans le classicisme et le romantisme allemands.

Dilthey a vu l'effet du mouvement romantique à travers trois réalisations valables. D'abord, l'exposé descriptif de Schiller sur la créativité esthétique en tant que corrélation active et réciproque entre la vie et la forme, l'expérience vécue et la forme artistique. «La loi de Schiller », — c'est ainsi que Dilthey nomme cette réciprocité, - en vigueur à travers Schelling jusqu'au dernier système de Hegel, a cependant brouillé la distinction essentielle entre l'appréciation esthétique et la compréhension philosophique. La deuxième de ces réalisations a été l'analyse kantienne du plaisir esthétique, indépendant d'un intermédiaire conceptuel, dont il résultait que la beauté ne pourrait plus être considérée comme une incarnation du «vrai». Kant a libéré la fonction de

12. Voir la nouvelle et troisième édition de cet ouvrage massif et complet: DILTHEY, W., Das Leben Schleiermachers, Berlin, éd. M. Redeker, 19661970, qui constitue les tomes XIII ${ }_{1}$ (1970), XIII $_{2}$ (1970), XIV ${ }_{1}$ (1966) et $\mathrm{XIV}_{2}$ (1966) des Oeuvres complètes (GS). Gadamer a appelé cet ouvrage «le seul classique dans la pratique herméneutique» (communication privée, été 1970). Pour la poétique de Schleiermacher, voir Werke, Berlin, éd. Jonas et d'autres, 1835-1864, la troisième partie du tome VII (Vorlesungen über die Aestbetik, 1842, — ce sont des notes de lecture de 1832-1833), les tomes XIII et IX (Literarische Nacblass, 1845 et 1849), et les cours plus anciens de 1819 et 1825 qui peuvent être trouvés dans Scbleiermachers Aestbetic, Berlin 1931, éd. R. Odebrecht. L'importance de l'œuvre de Schleiermacher pour la théorie de l'interprétation est indéniable, et l'absence d'une édition critique de ses cuvres aussi bien que d'une étude approfondie de son esthétique est regrettable. Néanmoins, voir WELLEK, $R$., «Schleiermacher », dans A History of Modern Criticism : 1750-1950, II, New Haven, 1955, pp. 303-308. 
la sensation dans la théorie esthétique d'une surdépendance à des primautés conceptuelles erronées. La difficulté, ici, a été l'incomplétude de l'analyse kantienne. Dilthey a voulu explorer la fonction sensitive dans tout le champ de la vie affective. La troisième réalisation a été l'accent que Herder a mis sur l'historicité de l'expérience esthétique, en opposition à l'universalisme de Kant et de Schiller. L'idéal de beauté et le goût se modifient nécessairement avec le changement des conditions historiques. Le problème, ici, a été l'insensibilité de Herder à la possibilité de coexistence de certaines caractéristiques universelles et de telles normes d'un conditionnement historique nécessaire à l'esthétique. ${ }^{13}$

Derrière ces trois réalisations, que Dilthey a identifiées avec Schiller, Kant et Herder, on retrouve l'idée d'un contrôle méthodologique, «...l'intention d'établir une relation serrée entre la constitution intellectuelle et spirituelle de l'artiste et son travail, c'est-à-dire entre la faculté créatrice et son produit, l'œuvre créée » (p. 77). Toutefois, res réalisations ont été obscurcies par l'orientation métaphysique qu'a prise l'idéalisme et qui aboutit aux confusions illuminantes de la théorie romantique. Dans sa propre analyse de la théorie littéraire, Dilthey a substitué à ces réalisations un exposé historique et phénoménologique de la vie humaine en tant que succession naturelle, tout en rétablissant la continuité de la théorie esthétique avec les thèmes interrompus de son passé.

\section{III — La thèse cardinale de la théorie diltbeyenne}

Si tel est le dialogue que Dilthey entretient avec la tradition postRenaissance, quel est le caractère spécifique de l'approche de la théorie littéraire que Dilthey adopte lui-même?

L'approche fondamentale de Dilthey vise à préciser la distinction traditionnelle entre les approches extrinsèques (biographiques, sociologiques, historiques) et les approches intrinsèques (l'œuvre elle-même considérée comme un univers autonome). Dilthey accepte l'argument de critiques formalistes contre les

13. Sur Kant et Schiller, voir Wellek, ibid., I, 227-257; et sur Herder, voir Wellek, ibid., I, 176-201, mais spécialement Sprachpbilosopbiscbe Scbriften, Hambourg, 1960, éd. Heintel. 
approches extrinsèques, mais il rejette la position formaliste ellemême en faveur de sa propre thèse. Cette thèse s'énonce comme suit: «...la nature de l'œuvre littéraire même ne peut pas être déterminée sans une recherche antérieure de sa position dans sa relation avec le monde de l'expérience humaine » (p. 81).

Comment Dilthey justifie-t-il cette thèse? Voici l'exposé de Müller-Vollmer: «(A) Si le poète présente, dans son œuvre, un monde indépendant, ces énoncés ne peuvent être vrais qu'en relation avec le monde poétique. (B) Les énoncés poétiques participent donc à la notion générale de vérité; en d'autres mots, ils ne peuvent pas signifier différemment des énoncés ordinaires. (C) S'il n'en était pas ainsi, les énoncés poétiques, à cause de leur manque d'un référent commun, ne pourraient pas être compris. (D) Ainsi donc, pour déterminer le caractère de vérité des énoncés poétiques, i.e. pour comprendre ce que signifie vraiment « la structure des signes » ou le Bedeutungsgefüge, nous devons mettre la vérité poétique en relation avec d'autres vérités que nous avons expérimentées. 》(P. 81.)

Il me semble que cette argumentation n'établit pas ce qu'elle devrait établir, parce que si l'énoncé (A) est une prémisse indéniable, l'énoncé (B) est extrêmement confus. Le résultat, c'est que l'énoncé $(C)$ est déplacé. Finalement, la conclusion ne suit pas.

La «notion générale de vérité » est tout simplement une caractérisation trop vague de la catégorie à laquelle la «vérité poétique est sensée appartenir ». En outre, la conjonction implicite (en B) de la vérité des énoncés poétiques et de la vérité des énoncés ordinaires, de leurs significations réciproques, est une double confusion. Premièrement, ces conjonctions rendent équivoques les termes «vérité 》 et «signification»; deuxièmement, ces conjonctions posent ( en d'autres mots») une équivalence entre la vérité de certains énoncés et la signification des mêmes énoncés. Mais il existe une distinction fort importante entre la vérité d'un énoncé et sa signification; cette distinction est déjà clairement établie dans la première édition de Logische Untersucbungen (1901) de Husserl dont Dilthey s'est servi à plusieurs reprises dans ses séminaires. 
Essayons donc de reconstruire cette argumentation. Voici une des possibilités de reconstruction.

(1) Pour déterminer la nature de tout système de signes, il faut faire appel à un référent extérieur à ce système, (2) parce que, si la nature d'un système de signes n'était qu'une fonction de son propre référent, elle serait indéterminable pour quiconque ne serait pas déjà familiarisé avec ce système. (3) Mais « l'œuvre littéraire même » est un système de signes. (4) Donc, pour déterminer la nature de «l'œuvre littéraire même », il faut faire appel à des facteurs extérieurs à l'œuvre d'art.

Cette argumentation, il me semble, est intéressante, mais, comme elle se présente ici, elle reste insatisfaisante. La raison en est que nous ne sommes pas certains de notre façon de comprendre le terme clé «système de signes». Il est évident que nous pouvons appeler certaines choses des «systèmes de signes 》, parce qu'en effet nous le faisons. Par exemple: les signes routiers, le sémaphore, l'algèbre booléenne, «The Solitary Reaper» de Wordsworth, la langue française. Mais il est également évident que tous les termes de cette énumération ne sont pas tous dans le même sens des «systèmes de signes 》. Nous ne savons donc pas clairement si la justification proposée pour (1), à savoir (2), est nécessaire et satisfaisante, parce que l'argumentation ne spécifie nulle part comment elle définit le «système de signes ». En fait, il se peut que certains systèmes de signes (par exemple, en phonologie, un ensemble particulier d'universels linguistiques) aient une telle portée que le besoin de recourit à un discours extérieur à ce système soit écarté. Dans tous les cas, l'argumentation que propose Müller-Vollmer pour supporter la thèse importante de Dilthey n'est pas pertinente. Ce que cette thèse demande vraiment, c'est que nous avancions plus loin dans la théorie de Dilthey.

\section{IV - La classification des énoncés}

Dans le cadre de son souci d'éviter les limitations d'un point de vue intrinsèque de l'œuvre d'art, Dilthey redéfinit sa tâche comme une double interrogation: les «principes de validité universelle » qui peuvent fonctionner normativement sont-ils valables pour les artistes et les critiques, et, s'ils le sont, quelles 
sont les relations avec les différents styles, techniques, et formes dans l'histoire? (p. 82). Ces deux interrogations se rapportent à un seul problème, la possibilité de faire des énoncés universellement valides. Pour résoudre ce problème, Dilthey entreprend une double démarche: il classifie les énoncés critiques comme des énoncés normatifs ; ensuite il recherche des énoncés normatifs universels. Cette double démarche échoue. En fait, Dilthey ne réussit pas à résoudre le problème qu'il soulève.

Dans son Einleitung in die Geisteswissenschaften (1883), Dilthey distinguait trois types d'énoncés, chacun relié à un différent type de position noétique : 1) l'assertion, 2) l'énoncé théorique, 3) l'énoncé pratique. Le premier type a trait à la perception des faits, par exemple, les énoncés historiques. Le deuxième type a trait à l'énonciation d'une relation entre des faits, par exemple, les énoncés psychologiques ou sociologiques. Et le troisième type a trait aux jugements de valeur, par exemple, les énoncés moraux ou éducationnels. Dilthey affirme ensuite qu'il peut y avoir une telle séparation parmi les positions reliées à ces énoncés, même si les différentes disciplines doivent employer un de ces types d'énoncés de façon prédominante.

Or, la théorie de la littérature, selon Dilthey, favorise les énoncés normatifs tout en impliquant non seulement des positions normatives mais aussi des positions factuelles et théoriques. Ce point nous renvoie à la critique fréquente de la position «historisante» de Dilthey. En effet, selon lui, la critique favoriserait le type d'énoncé utilisé par les théories d'éducation et d'éthique, en même temps qu'elle maintiendrait des énoncés historiques ou sociologiques.

Évidemment la difficulté de ces trois distinctions, c'est qu'elles sont grossières. On remarque immédiatement que les énoncés critiques sont aussi différents des énoncés éthiques ou éducationnels qu'ils le sont des énoncés historiques ou sociologiques. Ici, un développement n'est pas nécessaire ; les revues philosophiques bouillonnent encore d'activités dans ce domaine de la philosophie du langage. Mais la faiblesse de la classification diltheyenne des énoncés souligne l'importance du fait que l'élaboration d'une théorie adéquate des énoncés critiques est une des tâches que la théorie esthétique contemporaine n'a pas encore entreprises avec succès. 
Après avoir classifié les énoncés critiques comme des énoncés normatifs, Dilthey entreprend sa deuxième démarche, qui est la recherche d'énoncés normatifs universels «... sous un double aspect, à savoir la liaison des recherches concrètes dans les études culturelles et humanistes avec les analyses transcendentales des conditions qui ont tendu ces études possibles » (p. 87). Ce programme a été résumé dans l'exptession «l'historicisme transcendental de Dilthey», un mélange de la thèse de Vico sur l'intelligibilité intrinsèque de l'histoire humaine (l'homme peut comprendre l'histoire, parce que l'homme fait l'histoire) et de l'intérêt que Kant a porté à l'analyse des conditions qui rendent une expérience possible. ${ }^{14} \mathrm{La}$ théorie poétique de Dilthey devait fournir les fondements des conditions transcendentales de l'histoire humaine. En ce sens, la théorie poétique devait faire partie de l'élaboration d'une «critique de la raison historique» que Dilthey avait projetée, mais qu'il n'a pas réalisée.

Ces deux démarches, une théorie des énoncés et le concept de l'historicisme transcendental, sont le contexte de la méthodologie empirique que Dilthey a tenté de développer pour sa nouvelle théorie. La théorique était dans un état lamentable. L'herméneutique demeurait là où Schleiermacher l'avait laissée. Seule la linguistique comparative pouvait inspirer, parce que la linguistique était un domaine où Dilthey ne bénéficiait pas seulement d'amples connaissances, mais aussi de l'amitié de Lazarus et de Steinthal.

Toutefois, un des problèmes de l'utilisation de la linguistique dans la théorie littéraire était l'impossibilité, contrairement au domaine de la linguistique, d'élaborer une théorie générale de la littérature sur les bases d'un examen détaillé des aspects morphologiques et syntaxiques du langage. La critique doit pouvoir rendre compte de son expérience fréquente de la relation qui existe entre l'aspect intérieur d'un discours et sa forme linguisti-

14. Les théories de Kant sont célèbres, même si on en abuse. Pour une révision contemporaine, voir: Gianbattista Vico: An International Symposium, Baltimore, 1969, éd. G. Tagliacozzo, spécialement H. HODGES, «Vico and Dilthey » (pp. 439-447), H. READ, «Vico and the Genetic Theory of Poetry» (pp. 591-599), S. HAMPSHIRE, «Vico and the Contemporary Philosophy of Language 》 (pp. 483-497), et H.P. RICKMAN, «Vico and Dilthey's Methodology of Human Sciences» (pp. 447-457). 
que extérieure, une relation inobservable en linguistique. Les linguistes comme les critiques s'intéressent au même type d'objets, mais leurs points de vue sont différents. Le linguiste n'a qu'un intérêt générique pour les qualités particulières d'un phénomène individuel, alors que le critique s'intéresse aux mêmes qualités dans la mesure où il a eu une expérience vécue de leur individualité. La méthode comparative avait donc besoin d'être complétée.

Dilthey s'est alors tourné vers la nouvelle psychologie empirique de son temps dans l'espoir d'adapter une deuxième méthode empirique à son analyse du processus de création. Sa stratégie première a été de viser une nouvelle poétique avec le secours à la fois de l'analyse linguistique, de la poésie et d'une analyse psychologique de la poétisation (Dichten, le «poétiser»). Pratiquement parlant, Dilthey devait oublier la première et transformer la seconde en ontologie, son Lebensanalyse. De cette dernière émergea sa poétique. Finalement, la transformation reste regrettable. Dilthey ne fit que se perdre dans la tâche de reconstruire la psychologie de son temps, une psychologie encore très jeune. Les résultats auraient peut-être été plus probants aujourd'hui, s'il avait consacré un tel effort à la première démarche, plus tard oubliée, de sa stratégie. Il est clair qu'après tant de travail dans la philosophie des sciences sociales et dans la philosophie de l'esprit, plusieurs des spéculations génétiques de Dilthey apparaissent aujourd'hui comme une simple confusion. ${ }^{15}$ Mais l'inspiration de la linguistique a été ravivée. Et la critique structuraliste française n'est pas le seul exemple qui puisse être cité. ${ }^{16}$

15. Par exemple, voir: TAYLOR, C., The Explanation of Bebavior, Londres, 1964; ANSCOMBRE, E., Intention, Oxford, 1963 ; GEACH, P., Mental Acts, New York, 1964; FURLONG, E., Imagination, Londres, 1961. Cf. SARAWA, M., L'imagination selon Husserl, La Haye, 1970.

16. Sur le rôle de la linguistique dans la théorie littéraire contemporaine, voir: UITTI, K., Linguistics and Literary Theory, Engelwood Cliffs, 1969; HAMMOND, M., "Poetic syntax» in Poetics, La Haye, 1961; HENDRICKS, W., «Three Models for the Description of Poetry», Journal of Linguistics, V. (1969), pp 1-22; JACOBSON, R., "Linguistics and Poetics 》 in Sebeok, T. (éd.) Style in Language, Cambridge, Mass., 1960 ; LEVIN, S., Linguistic Structures in Poetry, La Haye, 1962 ; et THORNE, J.L., «Poetry, Stylistics and Imaginary Grammars », Journal of Linguistics, V, (1969), pp. 147-150. D'autres titres pourraient être ajoutés. 
Quels sont donc les éléments de la réflexion de Dilthey sur la genèse de la poésie?

\section{$\mathrm{V}$ - La genèse de la poésie}

Dilthey a d'abord essayé d'analyser les caractères distinctifs de l'imagination poétique en comparaison et en contradiction avec l'imagination commune. D'autre part, il a noté que, bien que l'imagination poétique soit foncièrement différente de l'imagination commune, elle était remarquablement semblable au type d'imagination que manifestent les rêveurs et les débiles mentaux. Pour pousser plus loin cette distinction, il a été forcé d'élaborer une théorie plus compréhensive que celles qu'avaient mises à sa disposition des théoriciens de la psychologie tel que Mundt. Cette tâche l'a amené à rejeter les hypothèses mécanistes de la psychologie behavioriste de son temps. portants.

Sa théorie peut être comprise à travers trois éléments im-

Dans son livre, une des réalisations particulières de MüllerVollmer est sa présentation du premier de ces éléments, le concept central et souvent obscur de cette théorie de Dilthey, le seelische Zusammenbang ou le continuum psychique. Parfois, Dilthey parle de ce continuum naturalistement et biologiquement comme d'un «fait empiriquement vérifiable » sous-jacent à tout phénomène mental. D'autres fois, il en parle psychologiquement comme d'une présence dans tout «processus formatif » donné qui conditionne les perceptions, les représentations et les sensations, une Gestalt psychique. Ailleurs, Dilthey décrit encore ce continuum ontologiquement comme une «...cristallisation de notre compréhension totale et de notre orientation dans le monde» (p. 110).

Se fondant sur ce concept, Dilthey veut insister sur le fait qu'un objet est toujours appréhendé dans une unité indifférenciée d'actes cognitifs, émotifs et volitifs, et non pas comme la perception d'un sujet connaissant particulier. L'unité de cette conscience multiple est une fonction de la Gestalt qui organise cette conscience. La Gestalt évalue le présent, interprète le passé, et annonce le futur. Cette unité organisatrice du continuum de la 
conscience $«$... est le produit de l'histoire et du développement personnel d'un individu, mais elle incorpore aussi, à un certain degré, les idées, les croyances, les façons de sentir et de penser qui prévalent à son temps et à sa place» (p. 113).

Le deuxième de ces éléments de la poétique de Dilthey est sa triple distinction des actes mentaux en représentations (Vorstellungen), en volitions (Willensaïsserungen) et en affections (Gefüble). Chacun des actes mentaux, tout en étant dans une certaine mesure tous les trois, est néanmoins plutôt d'un type en particulier. Chacun de ces types d'actes mentaux démarque une sphère particulière et, à l'intérieur de chaque sphère, Dilthey voudrait pouvoir distinguer un type d'imagination particulier. Ainsi, l'imagination scientifique, par laquelle Dilthey caractérise la capacité mentale de produire les hypothèses, appartient à la sphère cognitive. L'imagination pratique, par laquelle il caractérise la capacité mentale de produire des fins, des buts et des idéaux, appartient à la sphère volitive. Et l'imagination poétique, par laquelle il caractérise la capacité mentale de produire des images et des représentations de la vie, appartient à la sphère affective. Un état d'esprit affectif n'est proprement esthétique que lorsqu'il est distinct de toute activité volitive. Donc, «un état d'esprit esthétique... ne peut survenir que lorsque la projection de soimême vers un but futur ou une fin a cessé » (p. 117). Dilthey a également utilisé le terme «état d'âme» (Stimmung) pour dénoter cette conscience (awareness) sentie qui domine à la fois les représentations et les volitions. Dilthey poursuit donc en maintenant que de tels états d'âme esthétiques peuvent souvent engendrer des images particulières qui en retour transmettent le contenu affectif de cet état d'âme esthétique à d'autres personnes. L'imagination poétique est le pouvoir de produire de telles images.

Puisque l'affectif était le domaine de l'imagination poétique, Dilthey a cru qu'une analyse plus poussée de ce domaine pourrait jeter plus de lumière sur la nature de l'imagination poétique. Voici donc un troisième élément dans sa division du domaine affectif en un système de six sphères de la vie affective. Elles peuvent être énumérées comme suit : 1) les sensations physiques de plaisir ou de douleur (la chaleur) ; 2) les sensations reliées à des qualités sensorielles simples et à leur intensité (le son);3) 
les sensations qui proviennent de perceptions sensorielles différentes en relation (l'harmonie) ; 4) les sensations reliées aux actes cognitifs par lesquels ces relations sont effectuées (l'esprit, wit) ; 5) les sensations reliées à des conduites instinctives (la faim) ; 6) les sensations teliées à la conscience individuelle de potentialités (le courage). C'est à partir de ces six sphères de la vie affective que le poète formule un idéal de vie qui devient le centre d'une ouvre poétique.

Dilthey croyait que ces théories lui permettaient de distinguer comment le poète crée des images à partir du processus de production des images chez le rêveur et le débile mental. Tous les trois produisent des images, mais la Gestalt psychique acquise qui organise cette production n'est active que dans l'instance de l'activité poétique. Le poète ne perd jamais sa capacité de distinguer les images qu'il produit du monde de la réalité, alors que les images du rêveur et les hallucinations du débile mental se fondent souvent à leur conception de la réalité. La Gestalt psychique a un effet sur la relation entre l'imaginé et la réalité. En outre, elle structure l'unité artistique qui organise l'imaginé.

Dilthey croyait qu'une explication de la genèse psychologique de l'imagerie poétique aurait pu clarifier la nature de l'imagination poétique. Une image poétique était «... une représentation (verbale) d'une représentation (mentale)» (p. 133). Ces images étaient produites de la même manière que les images mémorielles l'étaient. Un état particulier de conscience contrôle l'expansion ou la réduction d'un état particulier de choses. Le même processus est mis en œuvre dans les anticipations du futur. Donc, la mémoire du passé et l'anticipation du futur dépendent, dans une certaine mesure, d'une transcendance du présent à travers la construction d'images mentales. Ce qui est distinctif de la construction poétique des images n'est pas le fait que les images verbales y soient substituées aux images mentales; c'est plutôt que les représentations originales n'en soient pas seulement soulevées ou contractées, mais qu'elles y soient aussi ajoutées. Toutefois, «l'addition de nouveaux éléments à la composition originale n'est pas un processus d'accumulation ou d'association. Ce sont plutôt les images elles-mêrnes, qui, lorsqu'elles émanent du noyau intérieur de l'acte créateur, transportent avec elles une énergie 
transmutationnelle infusée en elles par le jet de vie du poète» (p. 125). Dilthey veut alors poser que le processus poétique est caractérisé par «... le déploiement nucléaire des images sur le fond de la Gestalt psychique du poète, et sous l'impact d'un état affectif particulier ou 'état d'âme' » (p. 136).

\section{VI - L'expérience vécue et le concept du Type}

Dans une phrase célèbre, Dilthey supposait que l'art est «un organe pour la compréhension de la vie», si «la vie» est comprise comme le monde historique de l'homme. Le sens sousjacent de cette phrase est que l'art peut représenter des aspects essentiels de la vie humaine et devenir ainsi un moyen de comprendre le monde. Le sens ouvert en est qu'un contact avec l'art, aussi bien que des pensées scientifiques et philosophiques, est une ressource indépendante dans l'intérêt que l'homme porte à la compréhension de soi-même et du monde. Cependant, l'art est encore plus important que la science de la philosophie, parce qu'un contact avec l'art et la littérature élargit notre horizon de l'existence d'une façon différente de tous les autres types d'expérience. Car le poète peut représenter ce qui est essentiel à l'homme. L'essentiel est ici compris comme les valeurs qui nous sont révélées à l'intérieur de la conscience sentie des objets. Et la valeur est toujours comprise comme une fonction de la signification d'un objet particulier pour une vie individuelle en existence. Dilthey veut affirmer que «l'essentiel » ne peut pas être formulé dans des énoncés assertifs, parce qu'aucune forme assertive ne peut traduire la signification qu'un individu, par la réflexion, en est venu à attacher à certaines qualités de ses expériences propres. Seule la production poétique peut présenter «l'essentiel », ce qui est individuellement signifiant, parce que seuls les énoncés poétiques peuvent éviter les formulations assertives. Mais «l'essentiel » est toujours l'essentiel d'une expérience particulière vécue (das Eriebnis). Qu'entend alors Dilthey par Erlebnis?

Cinq sens de Erlebnis ont été distingués : 1) l'expérience personnelle de quelqu'un; 2) l'expérience intérieure de quelqu'un ; 3) une catégorie interprétative qui dénote un type particulier d'expérience ; 4) dans le sens de Erfabrung comme une expé- 
rience de tous les jours (Lebenswelt) ; ${ }^{17}$ 5) l'expérience immédiate par quelqu'un d'une signification générale.

C'est surtout le dernier de ces cinq sens qui prend ici de l'importance, parce que Dilthey maintient que la signification générale présentée dans une expérience immédiate ne peut pas être conceptualisée. L'œuvre d'art littéraire représente cette essence non conceptualisable de l'expérience vécue. La tâche du poète est de recréer un Erlebnis à travers l'imagination. Cet Erlebnis du poète ne peut cependant pas exister indépendamment de son expression artistique qui peut rester souvent incomplète. L'Erlebnis et l'œuvre d'art sont alors inséparables. «La nature de l'œuvre d'art littéraire, selon Dilthey, consiste dans l'articulation verbale expressive d'une expérience signifiante en un produit concret à travers lequel un aspect essentiel de la vie humaine est révélé »(p. 145).

Cette définition conduit au problème de l'universalité dans l'art. Comment l'expression d'une expérience particulière (Erlebnis) peut-elle exprimer en même temps quelque chose d'universel (Wesen) ? Dilthey $\epsilon$ ssaie de répondre à cette question en analysant comment «l'essentiel» (das Wesenbafte) est perçu. «L'essentiel » signifie, ici, « tout ce que l'individu existant perçoit et accentue comme étant caractéristique et nécessaire dans une relation vécue particulière...» (p. 146-147). Percevoir l'essentiel signifie percevoir ce qui est typique. Là où le scientifique et le philosophe produisent des concepts, le poète produit des types. Comme le Erlebnis, le typique a plus d'un sens dans le cours du développement diltheyen. Dilthey en emploie trois : 1) le sens ordinaire, quelques caractéristiques d'une personne etc.; 2) le sens scientifique, quelque chose appartenant à une classe définie ou à une espèce, etc.; 3) le sens ontologique, quelque chose nécessairement relié à l'existence.

Le dernier sens est encore le sens pertinent. Dilthey veut poser que «tous nos actes d'appréhension et de compréhension sont des actes typologisants avant de devenir conceptuels » (p.

17. Pour un compte rendu précis du concept ci-mentionné de Lebenswelt voir: BRAND, G., Die Lebenswelt: Eine Pbilosopbie des konkreten Apriori, Berlin, 1971. 
148). Dilthey parle alors d'un type de schématisation précon. ceptuel des impressions sensorielles. Cette relation nous amène à discerner deux nouveaux sens de «typique »: 4) un trait commun dans une variété de phénomènes ; 5) un cas idéal, un modèle ou une norme. Dilthey note maintenant que les types «idéels» sont spontanément produits par l'esprit. Il note encore que de tels types sont opératoires non seulement dans la perception mais aussi dans tout acte mental. En outre, ces types sont des représentations concrètes d'une signification essentielle d'un état particulier de choses. Ceci amène Dilthey à un nouveau et dernier sens de «type »: 6) une image concrète d'une représentation du général dans le particulier, — «l'essentiel dans le particulier ».

Mais comment percevoir l'essentiel dans le particulier? II faut comprendre que les «types» ne sont pas seulement des images préconceptuelles internes d'un monde extérieur.

Dilthey ne maintient que la dichotomie interne, - l'externe est inadéquate, - parce qu'un type émerge du contact entre un phénomène et la totalité de la conscience. Une représentation typique est l'unité entre le phénomène et la totalité vécue. L'artiste ne représente donc pas l'objectif; il représente le typique comme une convergence entre le phénomène et la conscience. Finalement, la solution diltheyenne au problème de la généralité et de l'universalité dans l'art fait appel à la présentation non discursive du sens par le symbole.

Comment Dilthey entend-il «le symbole»? Fondamentalement, de la même manière que Cassirer et Langer ${ }^{18}$, c'est-à-

18. CASSIRER, E., The Problem of Knowledge, New Haven, 1950, et The Pbilosophy of Symbolic Forms (3 volumes), New Haven, 1953-1957. LANGER, S., Pbilosopby in a New Key, New York, 1958; et Mind: An Essay on Human Feeling, Baltimore, 1969.

19. Voir l'édition et les notes de Steinthal de von HUMBOLDT, W., Die Sprachpbilosopbischen Werke, Berlin, 1884. L'influence centrale de von Humboldt sur les théories du langage de Heidegger peut être trouvée dans le dernier et le plus important chapitre du Unterwegs zur Sprache (Pfullingen 1959) de Heidegger. À ma connaissance, la meilleure présentation des théories de Humboldt est: BROWN, R., W. Von Humboldt's Conception of Linguistic Relativity, La Haye, 1967. Pour une présentation des théories complémentaires de B.L. Whorf, voir BLACK, M., "Linguistic relativity: the view of D.L. Whorf 》 Phil. Rev., IXVIII (1959), pp. 228-238. Sur deux 
dire, à toutes fins pratiques, comme Wilhelm Von Humboldt ${ }^{19}$. La liaison historique de Dilthey et de Humboldt provient des professeurs berlinois de Dilthey, l'historien Droysen et le linguiste Steinthal. Du point de vue de Humboldt, la médiation entre l'intérieur et l'extérieur est réalisée par le langage. Le langage n'est donc pas simplement objectivement orienté comme un moyen de transmettre l'information. Le langage est aussi subjectivement orienté comme un moyen de symboliser la réalité. Le langage se réfère à ces deux objets et exprime la pensée de celui qui parle.

Donc, pour Dilthey, le symbole est la fusion sensorielle de l'intérieur et de l'extérieur. Les symboles sont alors ambigus selon qu'on accentue leurs aspects référentiels ou leur aspect expressif. Et, indépendamment de notre façon d'interpréter un symbole, certains symboles sont basés sur la corrélation conventionnelle qui existe entre l'intérieur et l'extérieur. Ces conventions sont historiques. L'ambiguiité essentielle du symbole est donc d'être compris dans une double dimension : celle du présent intemporel (la corrélation conventionnelle) et celle du passé et du futur (l'histoire des corrélations conventionnelles). L'art peut alors être universel, parce que l'art «... est essentiellement symbolique, une excroissance de la faculté de production symbolique, qui est une partie intégrale de sa nature et fonde ainsi les racines du langage, de la mythodologie et de tous les arts 》 (p. 165). Cela signifie que l'art est universel d'une façon complexe, puisque son caractère symbolique peut fonctionner à la fois historiquement (en utilisant les symboles antérieurs) et créativement (en façonnant de nouveaux symboles). Il y a donc, pour Dilthey, deux sphères corrélatives de l'universalité : l'universalité du cosmos historico-culturel et l'universalité de la nature humaine.

Qu'est-ce qui distingue la symbolique littéraire? Dilthey répond. La symbolique poétique est nécessairement verbale. Cependant, la cohérence de cette symbolique verbale requiert à la

concepts centraux de la théorie de von Humboldt, voir: STOLTE, E., «W. von Humboldt's Begriff der Inneren Sprachform,» Zeits für Phon. und allg. Sprachwiss., II, (1948) 205-207; et WEISGERBER, L., «Zum Energeia Begriff in Humboldt's Sprachbetrachtung », Wirkendes Wort, IV, (1954), 374-377. 
fois une organisation temporelle et un ordre perceptible dans les diverses images employées. En outre, l'emploi du temps, de l'espace et de la causalité dans la symbolique poétique est distinctif, parce que la complétion est toujours réservée au travail de l'imagination des lecteurs. Le temps, l'espace et la causalité dans l'œuvre d'art littéraire ne peuvent pas exister indépendamment des interrelations entre des personnages et des événements fictifs. "L'œuvre littéraire ... prend son origine dans l'articulation d'un Erlebnis que le poète réalise au moyen des mots; il transforme alors cet Erlebnis en une cohérence totale d'images qui n'existe que dans son esprit et ceux du lecteur et de l'auditeur. » (P. 171.) ${ }^{20}$

Si tels sont les éléments importants de la poétique de Dilthey, est-ce qu'il y a des conclusions à tirer? Voici le témoignage de Müller-Vollmer. "Quoiqu'il en soit, Dilthey a montré de façon convaincante la nature historique de toute forme et technique littéraire et a ainsi démontré l'impossibilité de construire dorénavant une esthétique normative basée sur des lois intemporelles de la technique. 》 (P. 193.) Cette réalisation n'était pas le but original de Dilthey. Au départ, rappelons-nous, Dilthey avait espéré combler l'écart entre l'artiste et la critique en développant une théorie compréhensive qui aurait unifié à la fois l'art et l'esthétique. Ce but n'a jamais été atteint. Au contraire, les recherches auxquelles ce but a conduit Dilthey ont engendré un certain nombre de concepts qui ont renversé la prédominance de la théorie esthétique du XIXe siècle. Une question demeure cependant. Quels ont été les points valides de l'attaque diltheyenne de la théorie esthétique normative? En d'autres mots, quel argument, s'il en est un, Müller-Vollmer offre-t-il comme justification pour sa conclusion?

Le seul argument de Müller-Vollmer est le suivant : «Un symbole, selon Dilthey, incarne simultanément un type absolu

20. Pour un compte rendu philosophique récent de certains des problèmes que cette théorie de la symbolique littéraire ignore complètement, voir: $\mathbf{M}$. BEARDSLEY, "Metaphor, Encyclopedia of Pbilosopby (New York, 1967), V. pp. 284-288. Sur la théorie diltheyenne des éléments constitutifs des cuvres littéraires, il est intéressant de revoir les théories classiques. Par exemple: McCALL, M., Ancient Rhetorical Theories of Simile and Comparison, Cambridge, Mass., 1969. 
et un type relatif d'universalité. Étant l'expression d'une disposition fondamentalement humaine, un symbole est, dans un sens, méta-historique. Toutefois, cette disposition doit se réaliser dans des créations symboliques définies pour ne pas rester simplement potentielles. Par conséquent, le méta-historique existe pour nous dans ces diverses manifestations historiques et par elles. C'est pour cette raison qu'il ne peut y avoir des règles intemporelles régulatrices des formes et des techniques littéraires et artistiques. » (P. 195.)

Le noyau de cette argumentation est l'axiome qui, après Sartre, est pratiquement disparu de la littérature. Cet axiome est le suivant : il y a une nature humaine commune. Müller-Vollmer rapporte que cet axiome parcourt tous les écrits de Dilthey et qu'une partie de ses aspirations méthodologiques dans l'élaboration d'un historicisme transcendantal était de le rétablir (pp. 165-166). Mais un tel axiome ne peut être ni accepté ni refusé, parce que, tel qu'il se présente, il est notoirement ambigu. Nietzsche a attiré l'attention sur cette ambiguïté, bien avant Sartre, et les derniers écrits de Heidegger sont remplis de références à cette difficulté de définir cette ambiguïté plus clairement. En fait, la conclusion de Müller-Vollmer ne peut commander l'assentiment, parce que, comme la formulation de son problème, la conclusion reste trop vague.

Une partie de la tâche que Dilthey a laissée à la théorie littéraire contemporaine est le travail conceptuel de clarifier dans quel sens une œuvre littéraire réussie peut être à la fois historique et universelle et, plus fondamentalement, dans quel sens l'historicité textuelle fait-elle une élaboration «des normes objectives d'interprétation » nécessairement futile. C'est plus spécialement le deuxième de ces problèmes, "l'objectivité » des normes d'interprétation, qui est soulevé, dans sa totalité, par Müller-Vollmer, mais à la fin seulement de son enquête non critique sur la théorie littéraire de Dilthey. Au centre de ce problème réside la notion de «type », particulièrement dans ses relations avec la théorie, non satisfaisante, des énoncés. Ce qu'il reste à établir, en étendue et par plus d'un chercheur, c'est une théorie générale des énoncés normatifs qui couvrira non seulement les énoncés de la théorie littéraire, mais aussi l'énoncé des textes poétiques, et qui le fera 
précisément dans le contexte d'un emploi perspicace du concept diltheyen $\mathrm{du}$ 《type ».

Université d'Ottawa 